\title{
Surgery in South Africa - challenges and barriers
}

\author{
P Naidu, 1 (iD) I Buccimazza² (D) \\ ${ }^{1}$ Department of Surgery, Faculty of Medicine and Health Sciences, University of Cape Town, South Africa \\ ${ }^{2}$ Department of Surgery, Faculty of Health Sciences, University of KwaZulu-Natal, South Africa
}

Corresponding author, email: pnaidu2012@gmail.com

Background: Sustaining a surgical career can be challenging and there are numerous barriers to pursuing a career in surgery. These barriers and challenges are well reported in international literature, but there is a lack of knowledge on how this affects surgeons in South Africa. This study aimed to determine the barriers and challenges that South African surgeons face in their training and careers.

Methods: A 15-item questionnaire was designed and distributed via the Research Electronic Database Capturing software from 1 February-3 April 2020. Data were analysed in Stata 15 SE. All responses were anonymised.

Results: One hundred and twenty-nine participants responded to the questionnaire, $33(26 \%)$ of whom were female. The majority were specialist surgeons $(n=87 ; 71 \%)$. One hundred and eleven participants $(90 \%)$ reported they did not regret pursuing surgery. Barriers to pursuing surgery included limited personal time $(n=98 ; 76 \%)$, heavy surgical workload $(n=92 ; 71 \%)$, and difficulty taking leave of absence $(n=64 ; 50 \%)$, limited postgraduate training $(n=34 ; 26 \%)$, and verbal discouragement $(n=22 ; 17 \%)$. Challenges included difficulty maintaining work-life balance $(n=74 ; 56 \%)$, racial discrimination $(n=29 ; 23 \%)$ and gender discrimination $(n=15 ; 12 \%)$. Fifty-three per cent of participants experienced burnout.

Conclusion: Despite high career satisfaction, South African surgeons face numerous barriers to pursuing and challenges in sustaining a career in surgery and often experience burnout. These barriers and challenges disproportionately affect female surgeons and can be mitigated through formalised mentorship programmes, flexible work schedules, funding for postgraduate training, and training in diversity and discrimination.

Keywords: surgery, training, challenges, barriers

Appendix 1 available online: http://sajs.redbricklibrary.com/index.php/sajs/article/view/3391

\section{Introduction}

A career in surgery, while rewarding, is known to be highly competitive and demanding. ${ }^{1,2}$ The surgical work environment is challenging and fraught with mistreatment, which presents barriers to pursuing and difficulty in sustaining a surgical career. ${ }^{2}$

Reported challenges in the surgical workplace include discrimination on the basis of gender, race, pregnancy or childcare status; verbal, emotional, and physical abuse; sexual harassment; duty-hour violations; and genderbased salary discrepancies. ${ }^{3}$ These challenges perpetuate emotional and physical exhaustion and often deter medical students and junior doctors from pursuing a career in surgery. ${ }^{4}$ Overwhelming time commitments, lack of worklife balance, and disruption of personal life have all been cited as reasons for burnout, a common phenomenon among surgeons and surgical trainees., ${ }^{3,5}$ Nearly half the surgical resident workforce in the United States of America (USA) has reported burnout and one in 20 have reported suicidal ideation as a result of these workplace challenges. ${ }^{3,6,7}$ In sub-Saharan Africa, the demand on a surgical career is substantial owing to the high burden of surgical disease and lack of providers. ${ }^{8,9}$ These challenges may adversely affect the well-being of both patient and provider. ${ }^{10}$ In addition to burnout, surgeons often neglect their physical, spiritual and emotional health, and fail to seek medical attention following occupational injuries. ${ }^{10,11}$ Among female surgeons, higher rates of pregnancy complications and infertility have been reported. ${ }^{12}$

A surgical career can be particularly challenging for women who have to balance work schedules with pregnancy and motherhood, and work in a traditionally male-dominated field which can sometimes be hostile and unaccommodating. ${ }^{13}$ Female surgeons experience greater discrimination than their male counterparts, have greater barriers to pursuing a surgical career, and fewer opportunities for career advancement, particularly with respect to academia. ${ }^{13,14}$

While challenges such as long work hours and poor worklife balance are common across both genders, South African female surgeons face unique challenges. In a study by Roodt, one-third of participants, mostly male surgeons, felt that an increased number of female surgeons complicated or disrupted the departmental routine and the majority perceived males to be better suited to a career in surgery than females. ${ }^{4}$ Apart from this single-centre study in the Western Cape, there is a paucity of literature on the challenges of a surgical career among South African surgeons and trainees. 
The primary objective of this study was to evaluate the challenges of and barriers to pursuing a career in surgery amongst all surgical cadres throughout South Africa. A secondary objective was to establish whether some of these challenges and barriers are unique to female surgeons.

\section{Methods}

A 15-item questionnaire was designed on the Research Electronic Database Capture platform and included questions on both barriers to pursuing a career in surgery and challenges of a surgical career (as detailed in Appendix 1). The questions in the survey were informed by questions asked in previous studies, however, the survey was not a validated questionnaire or tool. Both male and female participants were asked questions on barriers and challenges, including questions on discrimination. The survey was circulated electronically to qualified general surgeons who are members of the Association of Surgeons of South Africa (ASSA) and/or Surgicom, and general surgical trainees/ medical officers through the various heads of surgical departments. Respondents could access the survey from 1 February-3 April 2020. Data were analysed in Stata 15 SE. All responses were anonymised and no identifying data were analysed. The ASSA committee approved the questionnaire for dissemination to its members. Ethical approval for this study was obtained (information removed for blinding). Descriptive analyses were performed as proportions, means and medians. Measures of association were performed

\begin{tabular}{lc}
$\begin{array}{l}\text { Table I: Demographic characteristics of respondents } \\
(\boldsymbol{n}=\mathbf{1 2 9})\end{array}$ \\
\hline Characteristics & $\boldsymbol{n}(\mathbf{\%})$ \\
\hline Gender & \\
Male & $96(74 \%)$ \\
Female & $33(26 \%)$ \\
Age & \\
\hline $20-29$ & $8(6 \%)$ \\
$30-39$ & $36(28 \%)$ \\
$40-49$ & $25(19 \%)$ \\
$>50$ & $60(47 \%)$ \\
Marital status & \\
\hline Married & $94(73 \%)$ \\
Single & $14(11 \%)$ \\
Non-marital relationship & $10(8 \%)$ \\
Divorced & $10(8 \%)$ \\
Other & $1(1 \%)$ \\
Training/practice location & \\
\hline Western Cape & $42(33 \%)$ \\
Gauteng & $41(32 \%)$ \\
KwaZulu-Natal & $24(19 \%)$ \\
Free State & $8(6 \%)$ \\
North West & $5(4 \%)$ \\
Eastern Cape & $3(2 \%)$ \\
Limpopo & $3(2 \%)$ \\
Northern Cape & $2(2 \%)$ \\
Mpumalanga & $1(1 \%)$ \\
\hline
\end{tabular}

using chi-squared tests. A $p$-value $<0.05$ was considered statistically significant.

\section{Definitions}

A specialist surgeon was defined as any surgeon who had qualified with a specialist degree in any surgical specialty and was currently employed as a consultant.

\begin{tabular}{|c|c|}
\hline Position & \\
\hline Specialist surgeon & $87(71 \%)$ \\
\hline Registrar & $23(19 \%)$ \\
\hline Fellow & $8(7 \%)$ \\
\hline Medical officer in surgery & $5(4 \%)$ \\
\hline Number of years in practice (median; IQR) & $18(10-28)$ \\
\hline \multicolumn{2}{|l|}{ Sub-specialty } \\
\hline No & $76(66 \%)$ \\
\hline Yes & $39(34 \%)$ \\
\hline \multicolumn{2}{|l|}{ Type of subspecialty } \\
\hline Colorectal & $9(23 \%)$ \\
\hline Gastroenterology & $7(18 \%)$ \\
\hline Breast and endocrine & $5(13 \%)$ \\
\hline Hepatobiliary & $4(10 \%)$ \\
\hline Trauma & $3(8 \%)$ \\
\hline Vascular & $3(8 \%)$ \\
\hline Bariatrics & $1(3 \%)$ \\
\hline Paediatric surgery & $1(3 \%)$ \\
\hline Transplant & $1(3 \%)$ \\
\hline Not specified & $5(11 \%)$ \\
\hline \multicolumn{2}{|l|}{ Type of post } \\
\hline Full-time public & $53(43 \%)$ \\
\hline Full-time private practice & $40(33 \%)$ \\
\hline Part-time public with RWOPs & $8(7 \%)$ \\
\hline Full-time private with public sessions & $7(6 \%)$ \\
\hline Part-time public & $4(3 \%)$ \\
\hline Employed abroad & $2(2 \%)$ \\
\hline Limited private practice & $2(2 \%)$ \\
\hline Other (retired/supernumerary/lecturer) & $7(14 \%)$ \\
\hline \multicolumn{2}{|l|}{$\begin{array}{l}\text { Reason for choosing surgery as a career } \\
{ }^{*} \text { participants were allowed to selected multiple options }\end{array}$} \\
\hline Technical aspect & $69(53 \%)$ \\
\hline Acute element of surgery & $66(51 \%)$ \\
\hline Inspired by role model & $49(38 \%)$ \\
\hline Academic competitiveness & $18(14 \%)$ \\
\hline Prestige & $8(6 \%)$ \\
\hline Family pressure & $2(2 \%)$ \\
\hline Other & $13(10 \%)$ \\
\hline \multicolumn{2}{|l|}{ Regret choice to pursue surgery? } \\
\hline No & $111(90 \%)$ \\
\hline Yes & $12(10 \%)$ \\
\hline
\end{tabular}




\section{Results}

One hundred and twenty-nine participants responded to the survey, $33(26 \%)$ of whom were female and $60(47 \%)$ were over the age of 50 years (Table I). The majority of respondents (84\%) were from the Western Cape, Gauteng, and KwaZulu-Natal. Specialist surgeons accounted for $71 \%$ of the responses $(n=87)$ and the median number of years in practice was 18 (IQR 10-28) (Table II). Most respondents were in full-time public practice $(n=53 ; 43 \%)$. The desire to pursue a surgical career was informed by a variety of reasons: technical aspect of surgery $(n=69 ; 53 \%)$, acute element of surgery $(n=66 ; 51 \%)$, inspired by a role model $(n=49 ; 38 \%)$, academic competitiveness $(n=18 ; 14 \%)$, prestige of surgery $(n=8 ; 6 \%)$, or family pressure $(n=2$; $2 \%$ ).

Many candidates perceived the demands of surgery, including a lack of personal time $(76 \%)$, heavy workload associated with surgery (71\%), and difficulty taking leave of absence $(50 \%)$, as potential barriers to a surgical career (Table III). More than half of the participants reported a lack of personal time to spend with family or for outside interests. Specific barriers to training included limited opportunities for postgraduate training $(26 \%)$ and the cost of training $(9 \%)$, as well as verbal discouragement (17\%) and a lack of mentorship (11\%).

Unique challenges of a surgical career related to a lack of a work-life balance $(57 \%)$, with $53 \%$ of participants reporting burnout. Specialist surgeons were more likely to experience burnout than any other position, although not statistically significant $(p=0.06)$. Other challenges related to a hostile work environment, including negative experiences with colleagues and discrimination and abuse of varying forms (Table IV). More participants reported negative experiences with male colleagues than with female colleagues $(p=0.02)$. In addition to challenges, candidates also suggested aspects that would facilitate surgical training, including formalised mentorship programmes (49\%), scholarships or funding to pursue postgraduate surgical training (47\%), and more flexible work schedules (36\%) (Table IV).

\section{Discrimination}

Both males and females were asked to report if they believed gender discrimination was a challenge in their surgical career; gender discrimination was reported as a challenge among 15 out of 33 females (45\%) and zero out of 96 males $(0 \%)$. Females were more likely than males to pursue surgery if inspired by a role model $(p=0.007)$ or due to family pressure $(p=0.015)$. With respect to barriers to pursuing a surgical career, $45 \%$ of females $(n=15)$ reported gender discrimination and were more likely than males to experience verbal discouragement $(p<0.001)$. Females more than males reported limited time to have a child $(p=0.002)$. Additionally, females were also more likely to report sexual harassment than males $(p<0.001)$. Female surgeons reported unique challenges including having to impersonate male qualities to toughen up $(n=11 ; 33 \%)$, not being treated equally to their male colleagues $(n=15 ; 45 \%)$, and a hostile culture toward females $(n=9 ; 27 \%)$.

Other forms of discrimination and abuse included racial discrimination $(n=29 ; 23 \%)$, physical abuse $(n=15 ; 12 \%)$, and sexual harassment $(n=7 ; 5 \%)$.

There were statistically significant differences in challenges reported by males compared with females

\begin{tabular}{lc} 
Table III: Barriers to pursuing a career in surgery $(\boldsymbol{n}=\mathbf{1 2 9})$ \\
\hline Limited personal time & $98(76 \%)$ \\
To spend time with family & $83(64 \%)$ \\
For outside interests & $70(54 \%)$ \\
To date/marry & $19(15 \%)$ \\
To have a child & $13(10 \%)$ \\
Surgical workload & $92(71 \%)$ \\
Difficulty taking leave of absence & $64(50 \%)$ \\
Limited postgraduate training & $34(26 \%)$ \\
Verbal discouragement & $22(17 \%)$ \\
Discouragement by male colleague & $15(68 \%)$ \\
Discouragement by female colleague & $7(32 \%)$ \\
Family aspirations & $11(9 \%)$ \\
Gender-based & $11(9 \%)$ \\
Age & $3(2 \%)$ \\
Other & $6 \%$ \\
Cost of training & $11(9 \%)$ \\
Lack of mentors & $14(11 \%)$ \\
Other (race/lack of experience/work & $10(8 \%)$ \\
environment) &
\end{tabular}

\begin{tabular}{ll}
\hline Table IV: Challenges of a career in surgery $(\boldsymbol{n}=\mathbf{1 2 9})$ \\
\hline Challenge & \\
\hline Difficult work-life balance & $74(57 \%)$ \\
Burnout & $68(53 \%)$ \\
Negative experience with colleagues & $47(36 \%)$ \\
Racial discrimination & $29(23 \%)$ \\
Gender discrimination & $15(12 \%)$ \\
Physical abuse & $15(12 \%)$ \\
Sexual harassment & $7(5 \%)$ \\
Impersonating male colleagues to toughen up & $11 / 33(33 \%)$ \\
Not treated equally to male colleagues & $15 / 33(45 \%)$ \\
Hostile culture toward females & $9 / 33(27 \%)$ \\
No challenges & $9(7 \%)$ \\
Negative experience with & \\
\hline Both & $26(57 \%)$ \\
Male colleague & $18(39 \%)$ \\
Female colleague & $2(4 \%)$ \\
Facilitation of surgical training & \\
\hline Mentorship programme & $76(59 \%)$ \\
Postgraduate surgical training scholarships & $61(47 \%)$ \\
Part-time surgical training programmes & $46(36 \%)$ \\
Other (flexible training programmes, & $17(13 \%)$ \\
appropriate cover of maternity leave, better & \\
working hours) & \\
\hline
\end{tabular}

(Table IV). While these challenges were reported by both males and females, female participants were more likely to report gender discrimination $(p<0.001)$, sexual harassment $(p<0.001)$, impersonating male colleagues to toughen up, not being treated equally to male colleagues $(p<0.001)$, and a hostile culture toward females $(p<0.001)$. 
The 12 candidates $(10 \%)$ who regretted their career choice did not face more gender discrimination $(p=0.154)$ or racial discrimination $(p=0.903)$ than those who did not regret their career choice.

\section{Discussion}

Our study highlights that substantial challenges and barriers exist within surgical training in South Africa. Burnout was reported among more than half of the participants. Challenges related to the demanding nature of a surgical career, heavy workloads and long hours, having limited personal time, and the lack of a work-life balance were most commonly reported. These challenges can further be exacerbated by bullying in the surgical environment which has previously been documented and defined as persistent negative behaviour or aggression, and is often experienced more by women. ${ }^{15,16}$ In our study, $17 \%$ of participants were verbally discouraged from pursuing a career in surgery, mostly by male colleagues and $36 \%$ of participants reported a negative experience, both of which can be considered as bullying. This form of mistreatment can be mitigated through training focused on professionalism.

In addition to bullying, other forms of discrimination were reported by our participants and can make sustaining a career in surgery difficult. Nearly one-quarter of participants experienced racial discrimination and $12 \%$ reported gender discrimination, forms of mistreatment also reported in international literature. ${ }^{15,17}$ Challenges and barriers were reported by both genders, but some were unique to and more common among females; for example, the need to emulate masculine qualities and a hostile culture toward females. Five per cent of participants experienced sexual harassment, and while this is lower than international literature, ${ }^{15}$ women reported sexual harassment more than men. Nearly half the female respondents reported gender-based discrimination and not being treated equally to their male colleagues. Additionally, female surgeons found having a child to be more challenging than males. The survey did not capture other forms of discrimination, such as religious or ethnic discrimination. These would be important to explore in more detail in future studies.

Despite these challenges, $90 \%$ of participants were satisfied with and did not regret their career choice. This is consistent with findings from previous studies. ${ }^{1,5,18}$ Some of the reported benefits of a surgical career in our study included job satisfaction, making a difference, and financial stability. While this study did not report the proportion of surgeons who wished to leave surgical practice, previous US-based studies have shown that despite high career satisfaction, one in four surgeons were considering leaving surgery on the basis of work-schedule demands and limited personal time. ${ }^{1}$

In comparison to a study published in the USA among residents, fewer South African residents reported gender discrimination $(31.9 \%$ in the US versus $21.7 \%$ in our South African study), fewer reported physical, verbal and emotional abuse $(30.3 \%$ in the US versus $17.4 \%$ in our South African study), yet more residents in South Africa reported burnout $(60.9 \%)$ in comparison to USA residents $(38.5 \%){ }^{3}$ Similarly, in internal medicine, a study conducted by the American College of Physicians reported that 51.3\% of female physicians reported gender discrimination, which is higher than that reported among our female cohort. ${ }^{19}$ In a study evaluating perceptions among residents in obstetrics and gynaecology, $40.6 \%$ of women and $2.9 \%$ of men reported gender discrimination, in comparison to our South African study in which $45 \%$ of female participants and $0 \%$ of male participants experienced gender discrimination. ${ }^{20}$ The challenges described in this study are not new or unique to South Africa or to the field of surgery; they are a global phenomenon and often disproportionately affect female surgeons. ${ }^{1,5}$ However, mitigating these challenges and barriers requires context-relevant local solutions. In South Africa, this could necessitate the restructuring of surgical training, including: i) a formalised mentorship programme; ii) programmatic changes that facilitate surgical training, such as scholarship opportunities for postgraduate training and part-time or more flexible work schedules; and iii) specific training in awareness of discrimination and accepting diversity in the discipline, particularly with respect to the mistreatment and challenges experienced by female surgeons.

Mentorship in surgery is gaining traction globally. ${ }^{21}$ Formalised mentorship programmes have been reported to aid job satisfaction and retention, facilitate career development, increase opportunities to engage in academia with respect to research and education, and improve technical skills and confidence. ${ }^{21,22}$ The goal of mentorship is to support and guide individuals through career and leadership development. The role of a mentor in career development can be substantial, allowing for expansion of professional network and providing a new personal or clinical perspective. Mentorship could be beneficial with regards to providing advice on how to achieve a work-life balance, or how to deal with or report discrimination. With a shortage of experienced faculty, the advent of online learning platforms and technology has proven to be useful in linking mentors and mentees. ${ }^{23,24}$ Exemplary role models who encourage diversity and inclusivity are crucial in changing the current hostile culture and biases in surgery. Previous studies have shown that medical students considering a career in surgery are more likely to be influenced by a positive role model than those who do not have such an influence. ${ }^{25}$ In our study, women were more likely to be inspired by a positive role model to pursue a career in surgery than men. Furthermore, access to career and academic opportunities were important motivators for choosing a career in surgery. ${ }^{26-28}$ Mentors can play an important role in career development, both in the academic and clinical settings. One-quarter of participants expressed there were limited opportunities for postgraduate training. More than half reported that mentorship programmes would facilitate surgical training. Further studies are required to address the value of mentorship and how this can be leveraged to create a more sustainable, inclusive, and safe environment for surgeons and surgical trainees, especially for women in surgery.

A study in the USA found that male surgeons were significantly less likely to encourage female medical students to pursue a career in surgery. ${ }^{1}$ In our study, females were more likely to experience verbal discouragement compared to their male colleagues, and this was cited as a barrier to pursuing a surgical career. This overt and implicit discouragement needs to be addressed if we are going to increase diversity and opportunities for minority groups, such as females, in the field of surgery. ${ }^{1,2}$ In recent years, there has been an increasing number of female medical students, with some medical schools reporting a female majority. ${ }^{29}$ If we continue to foster an environment that is 
hostile toward women in surgery, we will perpetuate the shortage of surgeons that currently exists. ${ }^{2,8}$

Furthermore, our study reported statistically significant differences in the challenges reported by males compared with females. Female participants were more likely to report gender discrimination, sexual harassment, impersonating male colleagues to toughen up, being treated unfairly compared with male colleagues, and a hostile culture towards female surgeons.

Professor Boffard, a world-renowned trauma surgeon and a past president of the International Society of Surgery, has been pioneering the way forward for female surgeons, being one of the first to recognise the need for and to implement part-time surgical training. These flexible work schedules are designed to "make surgery more attractive, particularly to women, who now consist of $60 \%$ of all medical graduates but only $5 \%$ of surgeons". ${ }^{29}$ However, in Johannesburg, more than half the surgeons are female and creating an environment that fosters gender equity is essential. ${ }^{29}$ More flexible work schedules will not only benefit female surgeons, but male surgeons too. The majority of our participants were male, and the majority reported that the surgical workload was a challenge to sustaining a career in surgery. Furthermore, more than half the participants reported burnout. The Flexibility in Duty Hour Requirements for Surgical Trainees (FIRST) Trial, conducted in the USA, has paved the way for the discussion on flexible work schedules in international literature, reporting that more flexible work schedules can decrease the notoriously high surgical workload, and assist in greater job satisfaction and career retention. ${ }^{30,31}$

South African surgical training lacks postgraduate programmes, mostly pertaining to fellowship opportunities. One-quarter of participants identified this as a challenge and nearly half reported a need for these programmes to facilitate surgical training. Postgraduate research fellowship grants and scholarships are available but limited, however not nearly as scarce as clinical fellowships. ${ }^{26-28}$ The ASSA Trust plans to source private funding to support fellowship posts. There is further opportunity to engage corporates, societies, and other organisations to fund postgraduate surgical fellowships, thereby encouraging continued learning in the field of surgery.

While our study focused mostly on gender discrimination, there is an opportunity to incorporate the value of diversity and the meaning of professionalism into formalised training curricula and to create a platform for both trainee and faculty surgeons to express their opinions, concerns, and suggestions about discrimination in the surgical workplace. By building intentional platforms for engagement into training, we will not only create awareness about any form of discrimination, but also help to decrease the biases in surgery. Non-technical skills are becoming increasingly important in surgical training and have been shown to facilitate cultural diversity and improved surgical outcomes. ${ }^{32,33}$ These existing platforms could be expanded to include training on the value of other types of diversity, including gender, ethnic and racial diversity.

\section{Study limitations}

This study was limited by its methodological design. The questionnaire was limiting, providing only quantitative answers. A mixed-methodology study design would have provided even more valuable and substantial information regarding the challenges of and barriers to a surgical career in South Africa. Additionally, this study was limited by the poor response rate, meaning that results are not necessarily generalisable to the entire South African surgical community. Despite these challenges, this study provides initial insight into the existing barriers to and challenges of a surgical career in South Africa. However, future studies should address the limitations discussed in this study.

\section{Conclusion}

This study highlights the numerous barriers to and challenges of surgical career in South Africa. More than half the South African surgeons who participated in this study experienced burnout. While barriers to pursuing and challenges in sustaining a surgical career are reported by both male and female surgeons, some inordinately affect females. Despite these challenges, career satisfaction was reported to be high among the overwhelming majority of surgeons. Encouraging sustainability of a surgical career requires addressing the barriers and challenges that exist. Potential solutions include formalised mentorship programmes, facilitating surgical training through postgraduate funding and flexible work schedules, and integrated training on discrimination and diversity. With increasing interest in medicine among females, the gender profile of medical school and postgraduate surgical training is changing. To address the shortage of surgeons, surgical work environments and training programmes need to adapt to reduce burnout and encourage inclusivity and diversity, especially among females.

\section{Acknowledgement}

We extend our gratitude to the steering committee of ASSA who enhanced the survey by their input, the ASSA secretariat for circulating it widely to the target audiences, and to all those who completed the survey.

\section{Conflict of interest}

The authors declare no conflict of interest.

\section{Funding source}

None.

\section{Disclaimer}

This survey was initiated by and conducted under the auspices of the Association of Surgeons of South Africa (ASSA).

\section{Ethical approval}

Ethical approval for this study was obtained from the University of KwaZulu-Natal Biomedical Research Ethics Committee (protocol number: BREC/00002259/2020).

\section{ORCID}

P Naidu (iD https://orcid.org/0000-0002-1112-9606

I Buccimazza (iD https://orcid.org/0000-0002-5399-3101

\section{REFERENCES}

1. Mahoney S, Strassle P, Schroen AT, et al. Survey of the US surgeon workforce - practice characteristics, job satisfaction, and reasons for leaving surgery. J Am Coll Surg. 2020 Mar;230(3):283-93. https://doi.org/10.1016/j. jamcollsurg.2019.12.003. 
2. Diaz A. Leaky pipelines and emptying wells - concerns from a survey of the US surgeon workforce. J Am Coll Surg. 2020;230(3):293-4. https://doi.org/10.1016/j.jamcollsurg. 2019.12.002.

3. Hu Y, Ellis RJ, Hewitt D, et al. Discrimination, abuse, harassment, and burnout in surgical residency training. $\mathrm{N}$ Engl J Med. 2019;18:1741-52. https://doi.org/10.1056/ NEJMsa1903759.

4. Roodt L. Female general surgeons: current status, perceptions and challenges in South Africa - pilot study at a single academic complex. [Thesis]. University of Cape Town, Faculty of Health Sciences, Department of Surgery, 2016. Available from: https://open.uct.ac.za/handle/11427/23661. Accessed 19 Apr 2020.

5. Dyrbye LN, Burke SE, Hardeman RR, et al. Association of clinical specialty with symptoms of burnout and career choice regret among US resident physicians. J Am Med Assoc. 2018 Sep 18;320(11):1114-30. https://doi.org/10.1001/ jama.2018.12615.

6. Wright A, Katz I. Beyond burnout - redesigning care to restore meaning and sanity for physicians. N Engl J Med. 2018;378(4):309-11. https://doi.org/10.1056/ NEJMp1716845.

7. Shanafelt T, Balch C, Bechamps GJ, et al. Burnout and career satisfaction among American surgeons. Ann Surg. 2009 Sep;250(3):463-71. https://doi.org/10.1097/SLA.0b013 e3181ac4dfd.

8. Kahn D, Pillay S, Veller MG, et al. General surgery in crisis - the critical shortage. S Afr J Surg. 2009;44(3):88-94. PMID: 16958234.

9. Chu K, Rosseel P, Gielis P, et al. Surgical task shifting in sub-Saharan Africa. Plos Med. 2009 May 19;6(5):e1000078. https://doi.org/10.1371/journal.pmed.1000078.

10. Brandt ML. Sustaining a career in surgery. Am J Surg. 2017;214:707-14.https://doi.org/10.1016/j.amjsurg.2017.06.0 22.

11. Yoo P, Tackett J, Maxfield M, et al. Personal and professional well-being of surgical residents in New England. J Am Coll Surg. 2017 Jun;224(6):1015-9. https://doi.org/10.1016/j. jamcollsurg.2016.12.024.

12. Anderson M, Goldman RH. Occupational reproductive hazards for female surgeons in the operating room - a review. JAMA Surg. 2020 Jan 2;155(3):243-9. https://doi. org/10.1001/jamasurg.2019.5420. Online ahead of print.

13. Kuehn BM. More women choose careers in surgery - bias, work-life issues remain challenges. JAMA. 2012;307:1899901. https://doi.org/10.1001/jama.2012.3725.

14. Cochran A, Hauschild T, Elder W, et al. Perceived genderbased barriers to careers in academic surgery. Am J Surg. 2013 Aug;206(2):263-8. https://doi.org/10.1016/j. amjsurg.2012.07.044.

15. Crebbin W, Campbell G, Hillis DA, et al. Prevalence of bullying, discrimination and sexual harassment in surgery in Australasia. ANZ J Surg. 2015;85(12):905-9. https://doi. org/10.1111/ans.13363.

16. Zhang L, Ellis R, Ma M, et al. Prevalence, types, and sources of bullying reported by US general surgery residents in 2019 . JAMA. 2020;323(20):2093-5.

17. Cochran A, Elder W, Crandall M, et al. Barriers to advancement in academic surgery - views of senior residents and early career faculty. Am J Surg. 2013 Nov;206(5):661-6. https://doi.org/10.1016/j.amjsurg.2013.07.003.
18. Tschuor C, Raptis DA, Morf MC, et al. Job satisfaction among chairs of surgery from Europe and North America. Surgery. 2014 Nov 1;156(5):1069-77.

19. Flint $\mathrm{JH}$, Jahangir AA, Browner BD, et al. The value of mentorship in orthopaedic surgery resident education - the residents' perspective. J Bone Joint Surg Am. 2009;91:101722. https://doi.org/10.2106/JBJS.H.00934.

20. Berlingo L, Girault A, Azria E, Goffinet F, Le Ray C. Women and academic careers in obstetrics and gynaecology: aspirations and obstacles among postgraduate trainees - a mixed-methods study. BJOG. 2019;126:770-7.

21. Kibbe MR, Pellegrini CA, Courtney MT. Characterisation of mentorship programs in departments of surgery in the United States. JAMA Surg. 2016;1:151(10):900-6. https://doi. org/10.1001/jamasurg.2016.1670.

22. Sinclair P, Fitzgerald JEF, Hornby ST, et al. Mentorship in surgical training - current status and a needs assessment for future mentoring programs in surgery. World J Surg. 2015 Oct 15;39(2):303-13. https://doi.org/10.1007/s00268-014-2774-x.

23. Healy NA, Cantillon MD, Malone C, et al. Role models and mentors in surgery. Am J Surg. 2012;204(2):256-61. https:// doi.org/1016/j.amjsurg.2011.09.031.

24. Program in global surgery and social change. Gender equity initiative. 2018. Available from: https://www.pgssc.org/ gender-equity-global-surgery. Accessed 7 Jun 2020.

25. Butkus R, Serchen J, Moyer DV, et al. Achieving gender equity in physician compensation and career advancement - a position paper of the American College of Physicians. Ann Intern Med. 2018;168:721-3. Epub 2018 Apr 17. https://doi. org/10.7326/M17-3438.

26. World without doctors [Internet]. Available from: https:// www.discovery.co.za/marketing/discovery-foundation-site/ grants.html. Accessed 4 Jun 2020

27. Scholarships and research grant funding | South African Medical Research Council [Internet]. Available from: https:// www.samrc.ac.za/funding/grants-and-scholarships. Accessed 4 Jun 2020

28. Funding emerging researchers [homepage on the Internet]. Available from: https://www.up.ac.za/hsc-research-funding/ article/1969290/research-funding-emerging-researchers. Accessed 4 Jun 2020

29. Boffard K. SA trauma expert to head world body. S Afr Med J. 2010;100:144-5.

30. Klingensmith ME, Awad M, Delman KA, et al. Early results from the Flexibility in Surgical Training Research Consortium - resident and program director attitudes toward flexible rotations in senior residency. J Surg Ed. 2015;72(6):e151-7. https://doi.org/10.1016/j.jsurg.2015.05.007.

31. Yang AD, Chung JW, Dahlke AR, et al. Differences in resident perceptions by postgraduate year of duty hour policies - an analysis from the flexibility in duty hour requirements for surgical trainees (FIRST) trial. J Am Coll Surg. 2017;224(2):103-12. https://doi.org/10.1016/j. jamcollsurg.2016.10.045.

32. Sharma B, Mishra A, Aggarwal R, Grantcharov TP. Nontechnical skills assessment in surgery. Surg Oncol. 2011 Sep;20(3):169-77. https://doi.org/10.1016/j.suronc.2010.10.0 01.

33. Waxman BP, Kitto SC, Willems A, et al. Interprofessional non-technical skills for surgeons in disaster response - a qualitative study of the Australian perspective. J Interprof Care. 2013;27:177-83. https://doi.org/10.3109/13561820.20 12.706337 . 